\title{
The Research of Light UAV Management Computer System
}

\author{
Li-qun Li and Hui Zhao \\ Aviation engineering institute, Civil Aviation Flight University of China \\ Sichuan Guanghan618307 \\ Llqun129@163.com
}

\begin{abstract}
This paper compiles system integrated control program based on MSP430F169 flight management computer design and production. There is interface circuit connecting with peripheral system in the flight management computer design. We implement the hardware connection of flight management computer with communication system, guided system, flight control system and power system by the interface circuit, by which realizes the both-way communication and autonomous flight of light UAV.
\end{abstract}

Keywords: Flight management computer, System integration, Interfacing, Data transmission

\section{Introduction}

Unmanned Aerial Vehicle (UAV) is a kind of driven by power, machine, reusable aircraft, Compared with manned aircraft, it has advantages of small volume, low cost, convenient to use. Unmanned Aerial Vehicle is mainly composed of ground control station and aircraft system, the ground control station including remote control handle, the power supply, the plane management computer, monitor, wireless transceiver module; Aircraft systems including aircraft body, power supply, the plane management computer, flight control, wireless transceiver module and navigation. The ground control station through wireless data transceiver module to send aircraft flight control, aircraft control according to the received data control aircraft rudder surface deflection control of the plane. Flight management computer is the core of the control system in the whole UAV (unmanned aerial vehicle). The proper functioning is very important for UAV control system. Flight management computer is the bridge of connecting every subsystem, by which it can be easy to realize the control of every subsystem [1-3]. This paper completed the system integration of hardware - based on MSP430F169 flight management computer and write to design and manufacture of systems integration control program. On the flight management computer designed for connected to the peripheral interface circuit. Through the computer and communication interface circuit to realize the plane management system, navigation system, flight control system, hardware interconnected power system. If there is breakdown in the system, we cannot implement data exchange of every system to cause that the whole UAV cannot be proper functioning. Thus, we should reduce the faulted probability to the greatest extent [4-5].

Flight management computer can make wireless both-way communication to do the transmission of control data, navigation data and other data. It offers technical support of follow-up autonomous flight. $433 \mathrm{MHZ}$ wireless communication module, can at the same time for 50 users to work simultaneously on the same occasion, without the user manual channel coordination and configuration. Receiving unit and the remote control unit has a key for code automatically function, can realize the two-way communication, digital address coding, large capacity, avoid address duplication, high sensitivity and strong anti-jamming capability. 


\section{Designing Scheme of Flight Management Computer}

The designing scheme includes two aspects. The one is main control chip. The other one is interface design.

The designing of flight management computer MCU selects multifunction ultra-low-power mixed signal to handle MSP430F169.

System integration establishes the physics connection between flight management computer and each system by interface circuit. For satisfying the need of system integration, we select UART for the communication interface of GPS model. UART interface is a kind of serial asynchronous communication. The transmit data rate is very slow. But the hardware requirement is low, and it is very easy to use. The hardware connections only need TXD, RXD and GND three lines [2].

UAV system integrates communication system, flight control system, guided system and other relevant system. For the normal running of every system control in flight management computer, every system control needs to assign MCU resource by time series. As shown in Figure 1, the sequential control diagram of system integration of unmanned aerial vehicle (UAV)

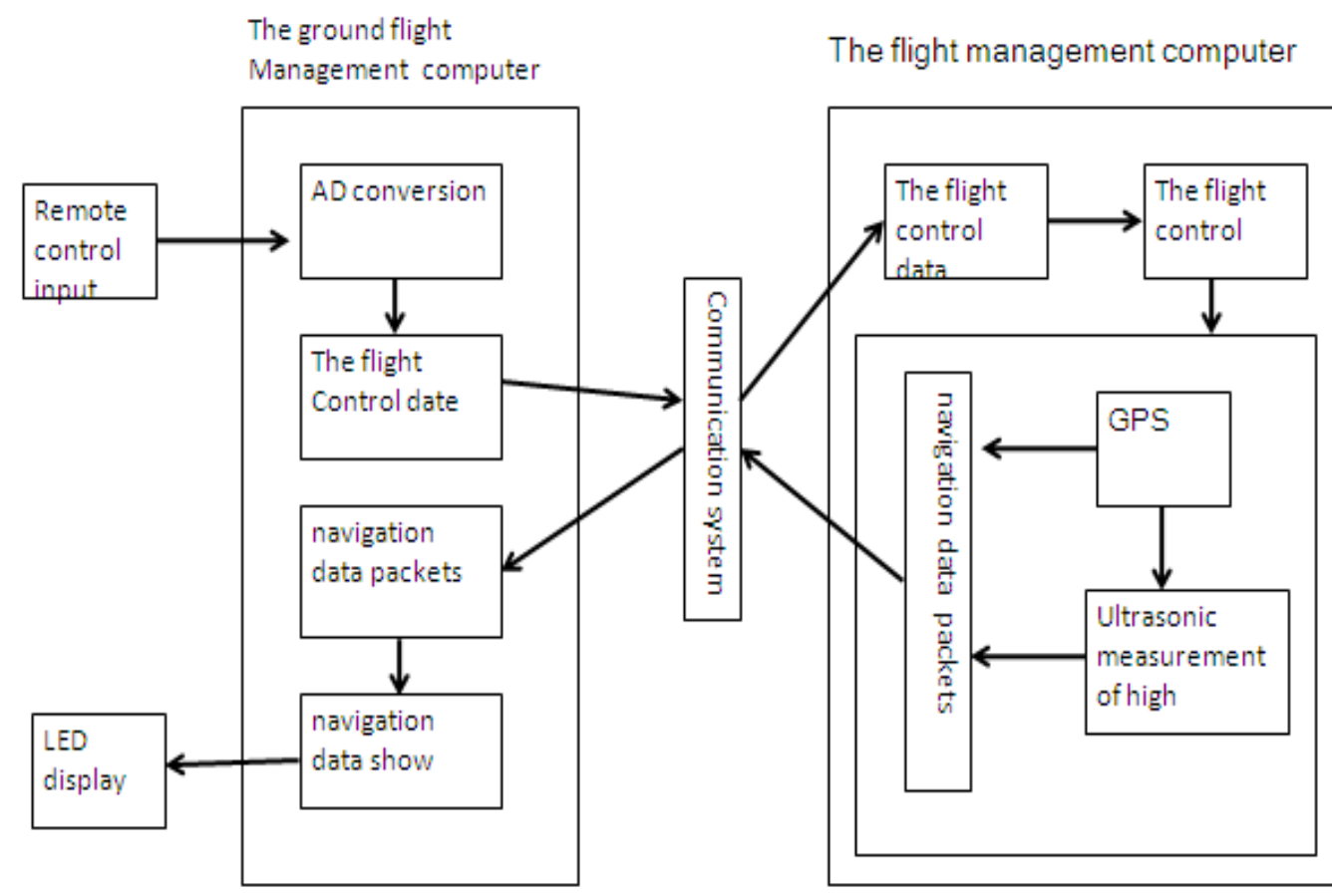

Figure 1. Sequential Control Diagram of System Integration

\section{Hardware Design of Flight Management Computer}

The flight management computer adoptive MCU is MSP430F169. As shown in Figure 2, Flight management computer hardware block diagram. 


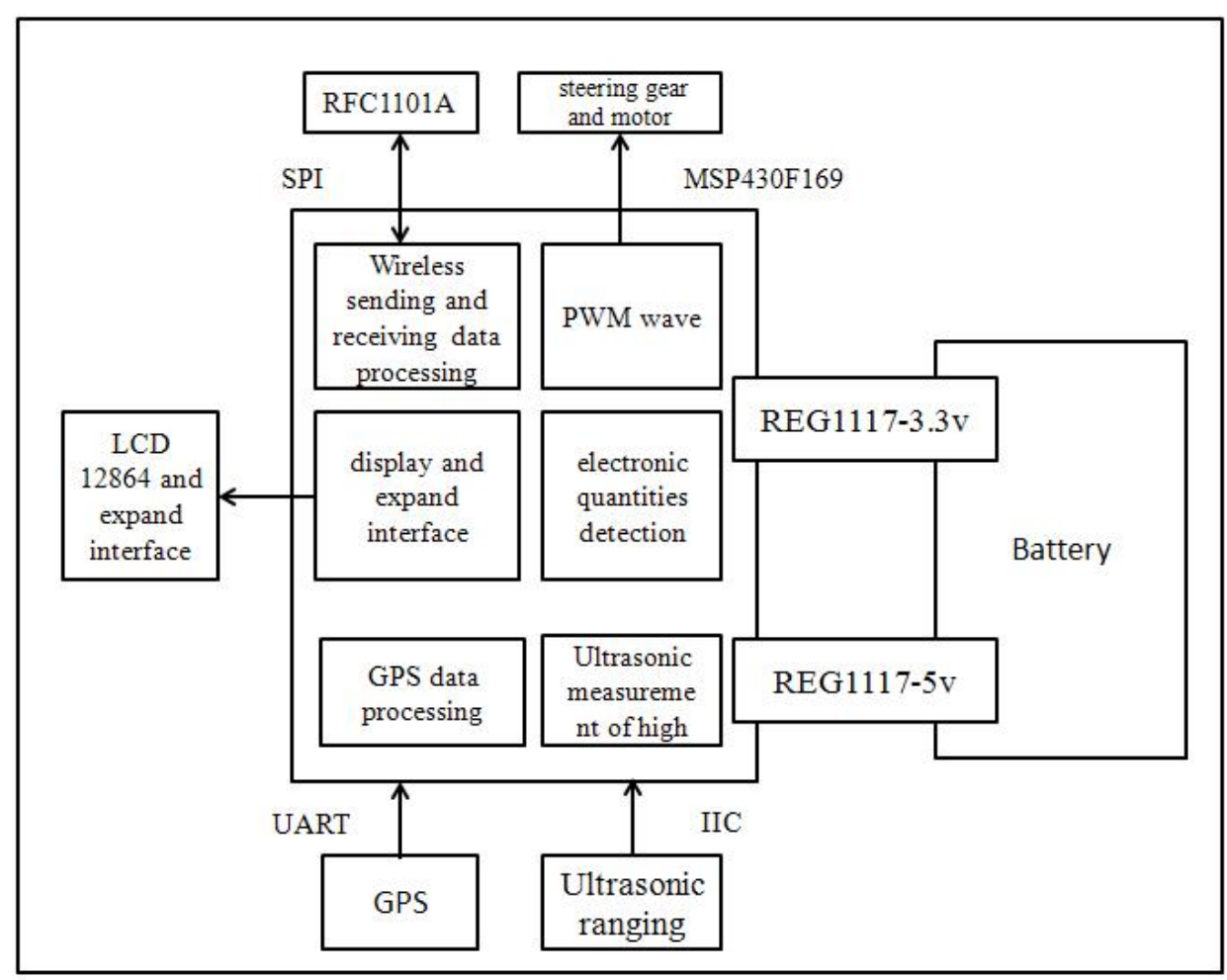

Figure 2. Flight Management Computer Hardware Block Diagram

The least system of MSP430F169 is the core of flight management computer. It includes the following parts, like MSP430F169 single chip, reset circuit, periphery clock circuit, JTAC simulated interface and BSL program download interface circuit. The MSP430F169 schematic diagram is like the Figure 3.

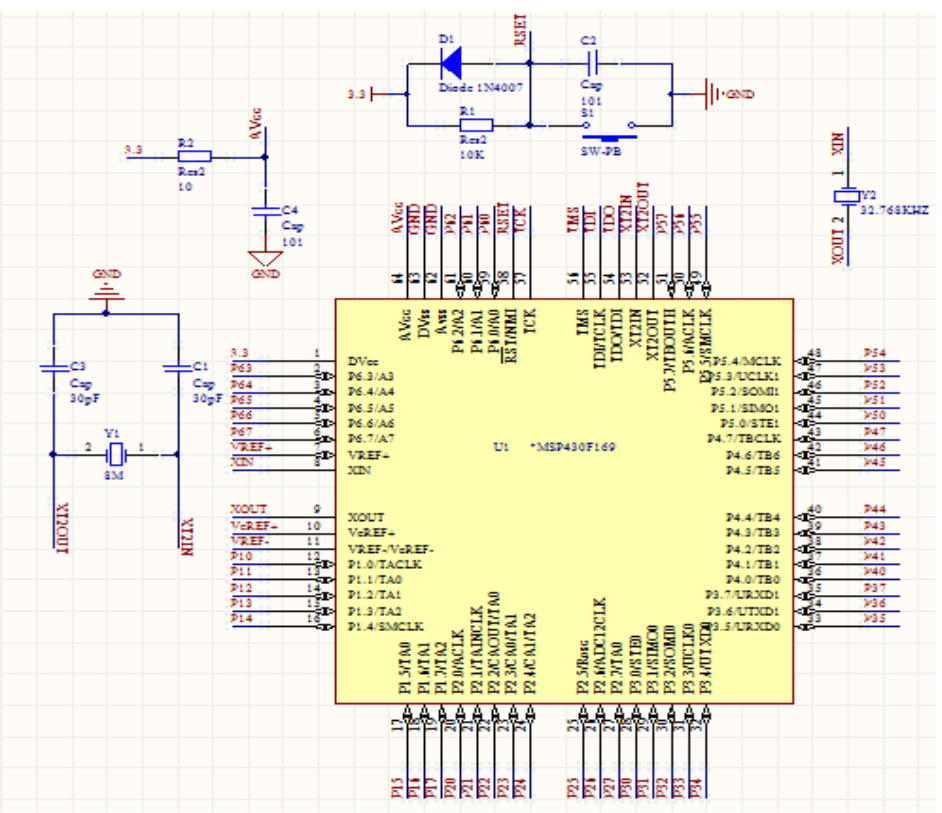

Figure 3. MCU Least System 


\subsection{Interface Circuit Design of Flight Management Computer}

The design of peripheral interface circuit is to realize the communication connection between MCU and peripheral model. It includes the following models' interface circuit, like SPI interface circuit communicated with RFC1101A model, UART interface circuit communicated with GPS model, IIC interface circuit communicated with ultrasonic model, four ways AD and PWM output interface and connected LCD and expansion interface circuits[7].

RFC1101A interface circuit is the bridge of establishing communication between RFC1101A wireless transmission model and MCU. Interface circuit is composed of SPI communication interface and RFC1101A control interface. The RFC1101A interface diagram's like the Figure 4.

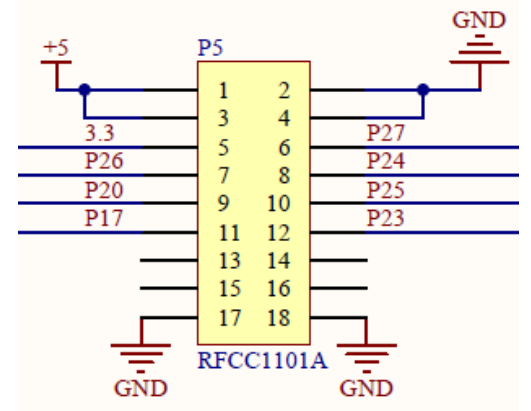

Figure 4. RFC1101A Interface Diagram

The communication between ultrasonic sounding model and MCU is implemented by IIC. Convenient I2C port is very easy to connect the wire. It only need connect VCC, GND, SDA and SCL four wires. High ultrasonic module interface circuit's like the Figure 5.

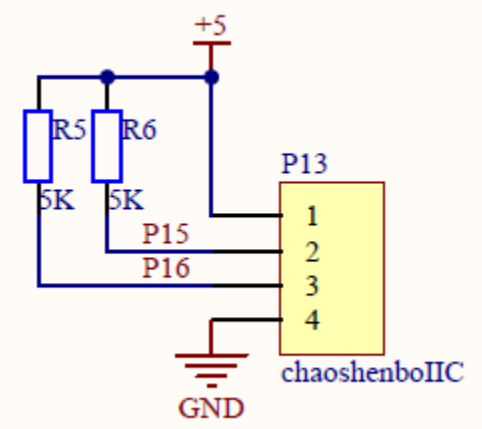

Figure 5. High Ultrasonic Module Interface Circuit

GPS model has abundant interface. The interface circuit design in flight management computer is the simplified design, which selects UART for reducing the complexity. GPS module interface is like the Figure 6. 


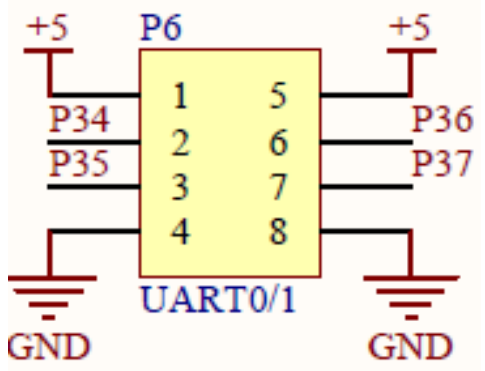

Figure 6. GPS Module Interface

This system adopts MSP430F169 AD model. The function of AD interface circuit in the ground is to collect voltage control signal from remote control handle input to finish control signal gathering. AD in the plane has been gathered by aircraft battery electronic detection input. Remote control and steering gear control input is like the Figure 7.
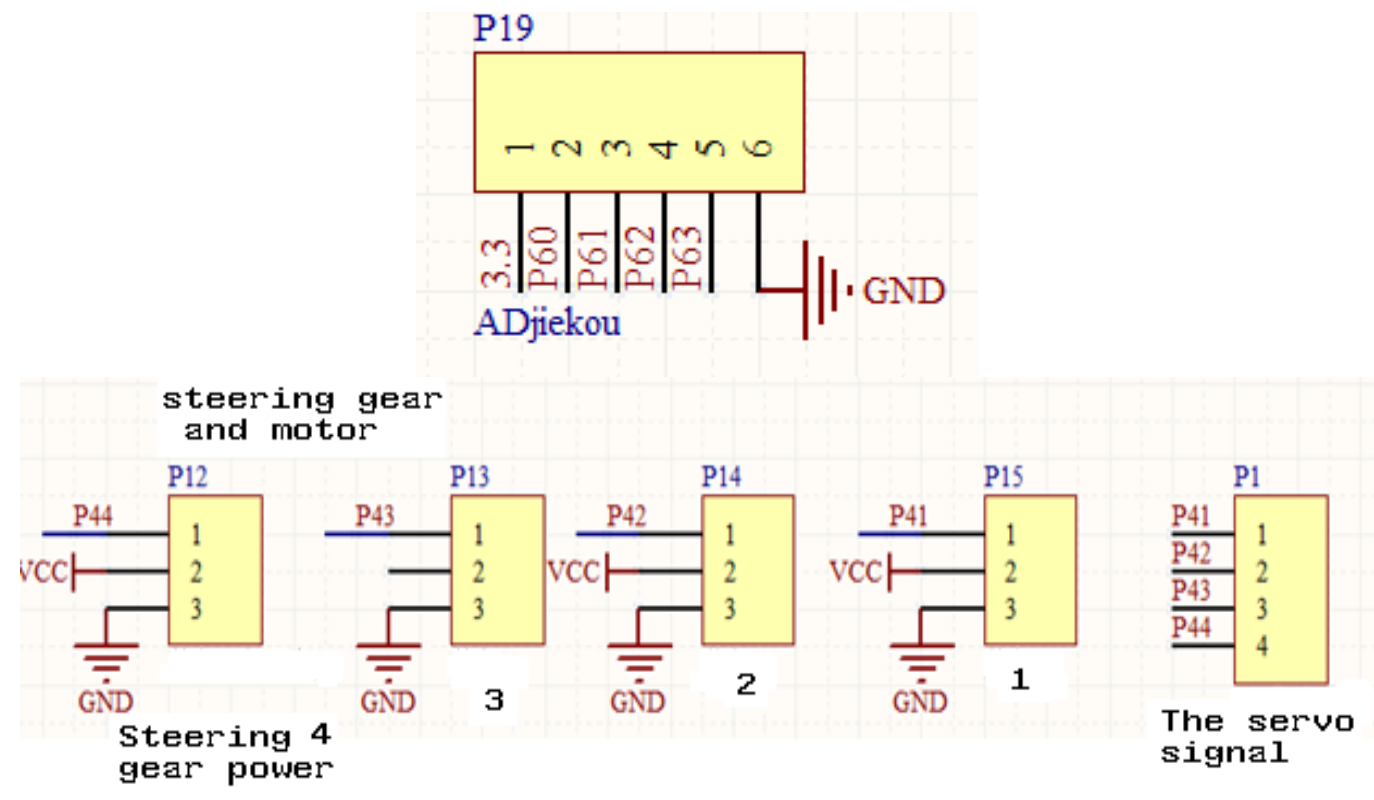

Figure 7. Remote Control and Steering Gear Control Input

LCD junction circuit selects LCD12864 as display. It is a raster graphics liquid crystal display model, which has multiple interface means like four bits/ eight bits parallel, two or three wires serial. The display resolution is $128^{*} 64$, in which has 8192 Chinese characters with the size of $16^{*} 16$ and 128 ASCII character set with the size of $16^{*} 8$. The flexible interface means and easy operational order can constitute friendly all Chinese man-machine interaction graphical interfaces. It not only can display $8 * 4$ rows Chinese characters with $16^{*} 16$ dot matrix, but also can finish graphical display. Low voltage and power dissipation is another outstanding feature. Compared with the same type graphical dot matrix LCD, the model constituted LCD is much more concise on hardware circuit configuration and display routine, and the price is just lower than the same dot matrix LCD. The interface circuit of LCD12864 is composed by five control lines, eight data lines and power line. 


\section{Software Design of Flight Management Computer}

MSP430F169 uses IAR Embedded Workbench EW430, which referred to as EW430 to program. EW430 not only supports 430's online simulation, but also can generate executable file for BSL burn.

\subsection{MSP430 Development Environment}

Preferable development environment is the essential for designing an integrated single chip system. EW430 is programming software with high office efficiency, which not only supports 430's online simulation, but also can generate executable file for BSL burn. The development tool has JTAG and BSL programming unit. For reducing the developing cost, we use BSL programming unit [8].

\subsection{Integrated Program Flow Chart of UAV}

UAV is composed by ground station and aircraft system. UAV programming is divided into two parts, like ground station programming and aircraft system programming according to the system constitution. The two parts has similarity in constituting framework. On the one hand, they both are compiled by modularized programming thinking. On the other hand, they both have wireless transmission programming block. And two programming both need to handle navigation data. The difference between them is that ground data is the display, but aircraft data is to resolve data for user.

There are two major functions about ground station. The one is to generate control signal and transmit it. The other one is to receive the signal and display navigation data and battery electric quantity message from aircraft. The programming involves AD transition, RFC1101A transmit-receive and inter conversion and LCD display.

Aircraft system has a few functions. The one is to receive control signal from ground and generate relevant PWM control wave. The other one is to get navigation data with periphery model and send packaged data back to the ground. The programming includes AD transition, RFC1101A transmit-receive, UART communication and navigation data analysis with GPS model and ultrasonic sounding IIC communication [9].

BSL burn software user interface of UAV system is concise and easy to use. We only need to add IAR programming software to generate executable file. And then we choose serial port and single chip type correctly to complete burn preparation. Finally, we can click the executable to start procedure burn.

\section{Debugging}

Main purpose of the system debugging is to verify communication condition between every subsystem and flight management computer and inspect whether each model runs well [10].

\subsection{Communication System Debugging}

Communication system is composed by RFC1101A wireless transmission model. Since it has no relevant simulated model in Proteus and CAD software, it cannot be emulated and debugged on PC. The debugging is to measure relevant pin bottom output waveform for working condition.

Flight management computer transceivers SI oscillogram in the ground station is like Figure 8 . The SO oscillogram in the aircraft station is like Figure 9. 


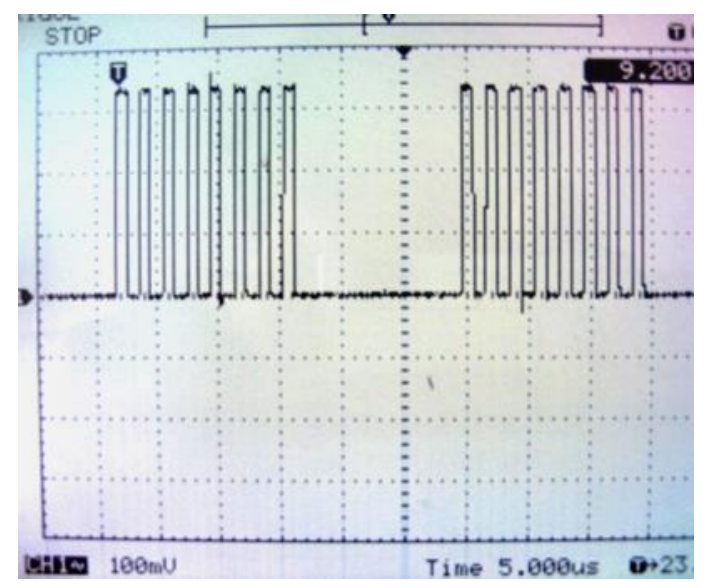

Figure 8. Ground Wireless Transmission SI Oscillogram

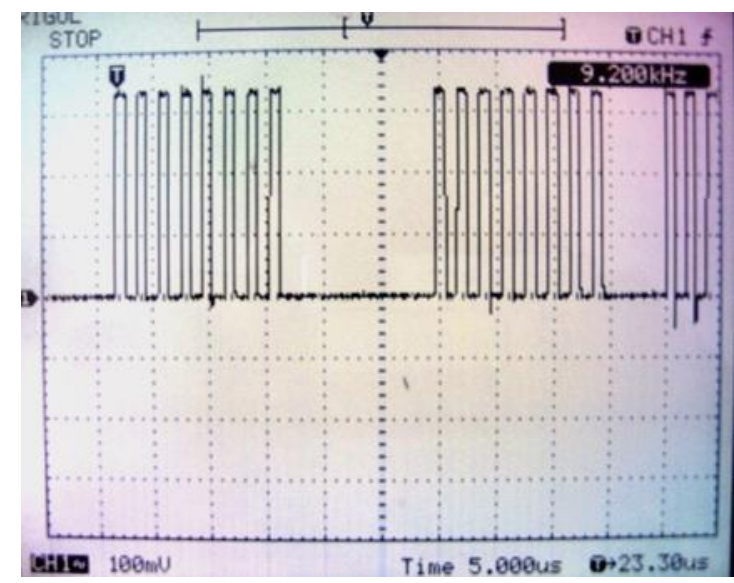

Figure 9. Aircraft Wireless Transmission SO Pin Transmission

After comparing the waveform of above two graphs, we can see that the waveform is basic unanimous and wireless transmission model run well.

\subsection{Navigation System Debugging}

Navigation system is composed by GPS and ultrasonic sounding. GPS debugging can be divided into PC debugging and flight management computer debugging.

Communication debugging between GPS model and PC is completed by serial debugging assistant. Firstly, we connect PC and GPS model by USB line. And then we open the serial debugging assistant on PC to set up serial. Baud rate is 9600. Check bit is NONE. Data bit is 8 . Stop bit is 1 . Then, we open the power the GPS model.

When it receives data from GPS model, LED2 would fast blink. When it correctly analyzes GPS navigation data, LED1 would blink.

Control system debugging. Control system debugging is waveform test of motor control output and four channels steering gear control output. Electromechanical control signal is PWM signal. After the test, we find that waveform got by receiving model and transmitter model are the unanimous. After rotating relevant controller, positive pulsing broadband of waveform would change. When potentiometer is in the different places, we measure the waveform like Figure 10-12. 


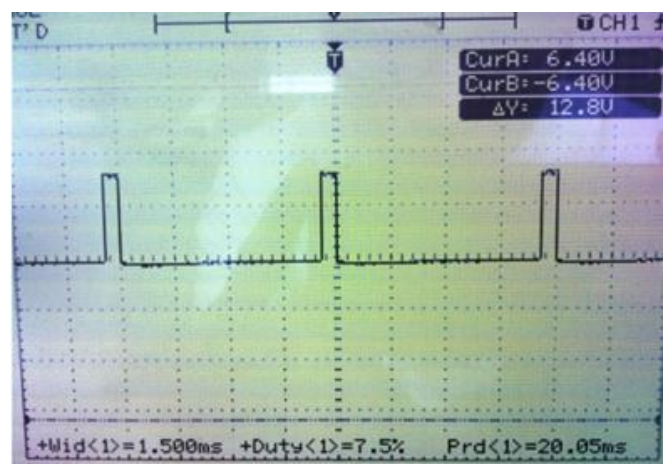

Figure 10. Potentiometer is in the Neutral Position

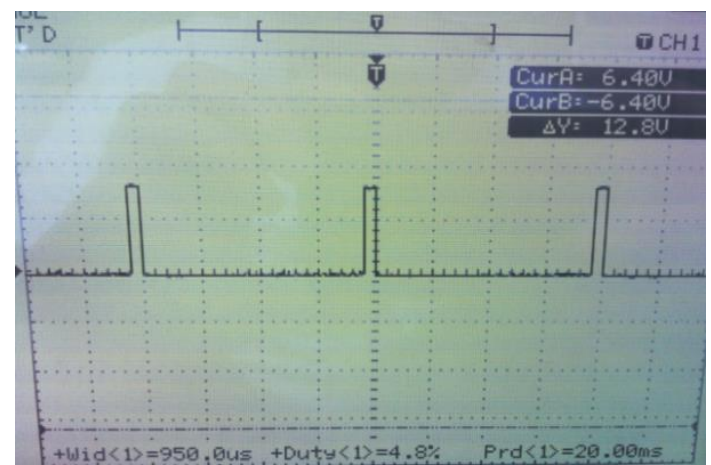

Figure 11. Potentiometer is in the Leftmost End

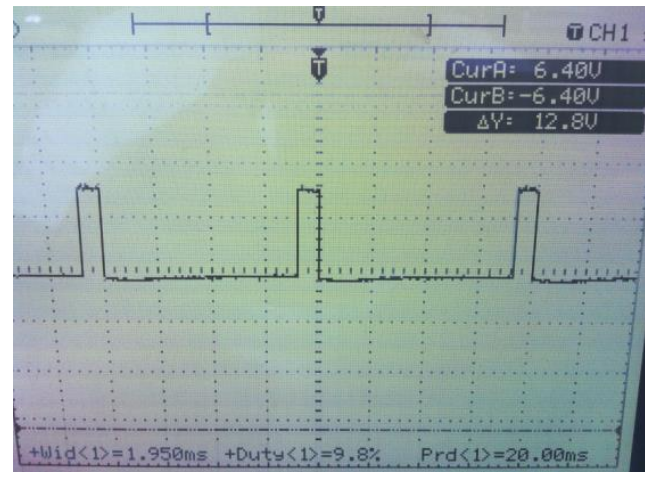

Figure 12. Potentiometer is in the Rightmost End

\section{Problems and Solution in Debugging}

In the verifying flight initial stage, system integrated autonomous UAV system appears serious electromagnetic interference problem. The problem is reflected between RFC1101A antenna, GPS antenna and servo mechanism. The major result of disturb is that engine body space is limited and adjustable of equipment layout is small. RFC1101A requires arrangement core surrounding. Thus, battery cabin and ultrasonic probe is close to RFC1101A. High frequency antenna should be far away from GPS antenna as far as possible in principle. We adopt the following measure. We readjust equipment layout to seek the optimum location of GPS antenna and high frequency antenna. And then we forward lead GPS antenna and retrude microwave antenna. 


\section{Reference}

[1]. J. Wiley \& Sons, "Unmanned Aircraft Systems -UAVS Design”, Development and Deployment Reg Austin, (2010).

[2]. Q. Li, S. Zhang and J. Huang, "Single chip microcomputer and port theory", Beijing: Electronic Industry Press, Beijing, (2008).

[3]. L. Ben, J. Eric and V. George, "Augmenting UAV Autonomy”, IEEE Robotics Automation Magazine, vol. 3, (2006), pp. 63-71.

[4]. D. Shim and H. Chung, "Autonomous Exploration in Unknown Urban Environment for Unmanned Aerial Vehicles", AIAA Guidance, Navigation and Control Conference, vol. 8, (2005), pp. 6381-6388.

[5]. K. Ahlstrom and J. Turin, "Future Architecture for flight control systems", Proceedings of the 20th Digital Avionics System Conference, vol. 10, no. 1, (2001), pp. 14-18, Beijing, china.

[6]. E. N. Wu, Q. A. Aydin, "Reliability-Based Modeling \& Analysis of Fault Tolerant Flight Control Systems, AIAA, (2005)

[7]. "Auto Pilot GCS Communication Protocol Manual”, UAV Flight Systems Inc., (2004).

[8]. “Texas Instruments Incorporated", MSP430F16X datasheet, (2011).

[9]. Hayward, Campbell and Larkin, "Radio-frequency circuit design actual book", M. Beijing: Posts and Telecom Press, (2009).

[10]. "Ground Pilot Ground Control Station System Manual, UAV Flight Systems Inc., (2004).

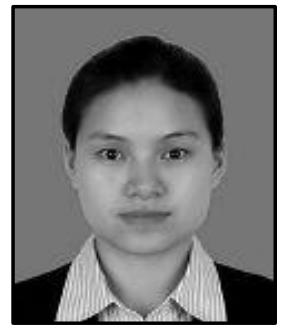

Li Liqun, female, born in 1983, master degree, experimentalist. The main research direction for aerospace electronics, electrical automation.

E-mail: $\underline{\text { llqun129@163.com }}$

Unit's name and Address: Aviation engineering institute, Civil Aviation Flight University of China, Sichuan Guanghan618307

Huizhao, female, born in 1970, master degree, engineer. The main research direction for Aircraft maintenance

E-mail: 1lqun129421@gmail.com

Unit's name and Address: Mianyang Sub-College, Civil Aviation Flight University of China, Sichuan Guanghan618307 
International Journal of Control and Automation Vol. 8, No. 2 (2015) 